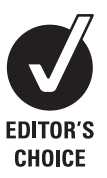

Melbourne Sexual Health Centre, Alfred Hospital and School of Population Health, University of Melbourne, Carlton, Australia; ${ }^{2}$ School of Population Health, University of Melbourne, Carlton, Australia; ${ }^{3}$ Centre for Molecular, Environmental,

Genetic and Analytic Epidemiology, School of Population Health, University of Melbourne, Carlton, Australia;

${ }^{4}$ National Centre in HIV Epidemiology and Clinical Research, University of New South Wales, New South Wales, Australia; ${ }^{5}$ Melbourne Sexual Health Centre, Alfred Hospital and Department of Epidemiology and Preventive Medicine, Monash University, Prahran, Victoria, Australia

Correspondence to:

Dr C K Fairley, Melbourne Sexual Health Centre, 580 Swanston

Street, Carlton, Victoria

Australia, 3053; cfairley@

unimelb.edu.au

Accepted 10 August 2009

Published Online First

16 October 2009

\title{
Rapid decline in presentations of genital warts after the implementation of a national quadrivalent human papillomavirus vaccination programme for young women
}

\author{
C K Fairley, ${ }^{1}$ J S Hocking, ${ }^{2}$ L C Gurrin, ${ }^{3}$ M Y Chen, ${ }^{1}$ B Donovan, ${ }^{4}$ C S Bradshaw ${ }^{5}$
}

\begin{abstract}
Objective: This study aimed to determine if the Australian human papillomavirus (HPV) vaccination programme has had a population impact on presentations of genital warts.
\end{abstract}

Methods: Retrospective study comparing the proportion of new clients with genital warts attending Melbourne Sexual Health Centre (MSHC) from January 2004 to December 2008. Australia provided free quadrivalent HPV vaccine to 12-18-year-old girls in a school-based programme from April 2007, and to women 26 years and younger through general practices from July 2007.

Results: 36055 new clients attended MSHC between 2004 and 2008 and genital warts were diagnosed in 3826 (10.6\%; $95 \% \mathrm{Cl} 10.3$ to 10.9$)$. The proportion of women under 28 years with warts diagnosed decreased by $25.1 \%$ (95\% Cl $30.5 \%$ to $19.3 \%$ ) per quarter in 2008 . Comparing this to a negligible increase of $1.8 \%(95 \% \mathrm{Cl}$ $0.2 \%$ to $3.4 \%$ ) per quarter from the start of 2004 to the end of 2007 also in women under 28 years generates strong evidence of a difference in these two trends $(p<0.001)$. There was no evidence of a difference in trend for the quarterly proportions before and after the end of 2007 for any other subgroup, and on only one occasion was there strong evidence of a trend different to zero, for heterosexual men in 2008 in whom the average quarterly change was a decrease of $5 \%(95 \% \mathrm{Cl} 0.5 \%$ to 9.4\%; $p=0.031$ ).

Conclusions: The data suggest that a rapid and marked reduction in the incidence of genital warts among vaccinated women may be achievable through an HPV vaccination programme targeting women, and supports some benefit being conferred to heterosexual men.

There are currently two vaccines for genital human papillomavirus (HPV) that have been licensed for use. One is the bivalent vaccine Cervarix (GlaxoSmithKline, Brentford, Middlesex, UK), which protects against HPV types 16 and 18, and the other is the quadrivalent vaccine Gardasil (Merck and Co Inc, West Point, Pennsylvania, USA), which protects against HPV types 6, 11, 16 and 18. HPV types 6 and 11 are associated with genital warts, whereas infection with types 16 and 18 is associated with high-grade squamous intraepithelial lesions of the cervix and other anogenital sites. Clinical trials have shown that both vaccines are highly effective at preventing persistent HPV infections and HPV-related squamous intraepithelial lesions of the cervix, ${ }^{1-7}$ while the quadrivalent vaccine has also been found to be highly effective at preventing genital warts in women ${ }^{8}$ and men $^{9}$ in clinical trials.

Governments around the world are currently deciding which vaccine should be included in immunisation programmes, and to do this robust data on the impact of the vaccine at a population level to demonstrate its effectiveness, rather than just efficacy, are required. The cost-effectiveness analyses being used by governments to inform these decisions currently rely on assumptions about the probable population impact of these vaccines because no population-level data are available. $^{1011}$ Policy decisions would be greatly strengthened by data from population-based vaccination programmes already in place.

Australia is in a unique position to provide such data. It was one of the first countries to implement $\mathrm{HPV}$ vaccination free of charge to women. It distributed the quadrivalent vaccine in April 2007 to girls in school, and in July 2007 to women less than 27 years of age outside of the school-based programme (http://www.health.gov.au/internet/ immunise/publishing.nsf/content/immunise-hpv/). Because Australia has achieved a higher level of coverage than other countries for women outside the school-based programme, it is likely to be the first to see significant changes in presentations of genital warts if they were to occur. Our aim was to determine if the national HPV vaccination programme has had a measurable impact on clinical presentations of genital warts at a large sexual health service in the year following its implementation.

\section{METHODS}

We conducted a retrospective study investigating the proportion of new clients who were diagnosed with genital warts attending Melbourne Sexual Health Centre (MSHC) between 1 January 2004 and 31 December 2008. MSHC is the major public sexual health clinic serving the city of Melbourne (population 3.5 million), Australia. MSHC provides a walk-in, triage service that prioritises access to the centre for clients who belong to risk groups or who have symptoms. ${ }^{12}$ Referrals are not required and services are free of charge. Genital warts are not a notifiable infection in any state or territory of Australia. In 2008, MSHC diagnosed $7 \%$ of chlamydia cases, $21 \%$ of gonorrhoea cases and $27 \%$ of syphilis cases in the state of Victoria (population 5.5 million).

Clinical and epidemiological data are routinely entered into the clinic electronic database by 
Table 1 Number (N) and percentage of clients diagnosed with genital warts by year and by risk group

\begin{tabular}{lcclccc}
\hline Characteristics & $\mathbf{2 0 0 4 - 7}$ & $\mathbf{2 0 0 4}$ & $\mathbf{2 0 0 5}$ & $\mathbf{2 0 0 6}$ & $\mathbf{2 0 0 7}$ & $\mathbf{2 0 0 8}$ \\
& $\mathbf{N}(\%)$ & $\mathbf{N}(\%)$ & $\mathbf{N}(\%)$ & $\mathbf{N}(\%)$ & $\mathbf{N}(\%)$ & $\mathbf{N}(\%)$ \\
\hline All & $3156(11.2)$ & $777(11.0)$ & $784(11.3)$ & $806(11.6)$ & $789(11.0)$ & $670(8.4)$ \\
All women & $1131(9.9)$ & $247(8.7)$ & $275(9.7)$ & $304(11)$ & $305(10.4)$ & $197(6.2)$ \\
Women $<28$ years & $850(12.7)$ & $177(11.1)$ & $205(12.4)$ & $242(14.6)$ & $226(12.7)$ & $130(6.6)$ \\
Women $\geqslant 28$ years & $281(6.3)$ & $70(5.6)$ & $70(6.0)$ & $62(5.6)$ & $79(6.8)$ & $67(5.4)$ \\
All men & $2024(12.1)$ & $529(12.6)$ & $509(12.5)$ & $502(11.9)$ & $484(11.5)$ & $473(9.9)$ \\
Men sex with men only* & $285(8.2)$ & $62(7.5)$ & $77(9.6)$ & $69(7.5)$ & $77(8.4)$ & $83(7.6)$ \\
Men sex with women only* & $1525(14.3)$ & $411(14.5)$ & $382(14.4)$ & $375(14.2)$ & $357(14.0)$ & $333(11.8)$ \\
Primary genital herpes & $501(1.8 \%)$ & $126(1.8)$ & $114(1.6)$ & $115(1.7)$ & $146(2.2)$ & $152(1.9)$ \\
No of clients seen & 28093 & 7051 & 6926 & 6968 & 7148 & 7962 \\
\hline
\end{tabular}

${ }^{*}$ Refers to the gender of the sexual partners in the past 12 months.

clinicians during every client consultation. These data included the diagnosis and the number and gender of sexual partners in the previous 12 months. The quadrivalent HPV vaccine was made available in Australia free of charge from April 2007 to girls aged 12 and 13 years in a school-based programme and to a catch-up group of 13-18-year-old girls in a largely school-based programme. From July 2007 it became available free of charge through general practitioners and community immunisation clinics for young women, aged up to and including 26 years. There were no programmes targeting men, although boys aged 9-15 years could be prescribed the vaccine privately at an outof-pocket cost of approximately US\$300. Data on the schoolbased vaccination programme in Victoria was obtained from The Department of Human Services Victoria (www.dhs.vic. gov.au). At the end of 2007 between $69 \%$ and $75 \%$ of girls in years 7, 10,11 and 12 (approximate ages of 12, 15, 16 and 17 years, respectively) had received three doses of the vaccine (Dr R Lester, personal communication). Coverage rates in women between 18 and 26 years of age are estimated by CSL Biotherapies to be $65-70 \%$ based on the number of doses of Gardasil distributed to general practitioners and community immunisation clinics in Victoria and the number of women in that age group living in Victoria (G Whiteside, CSL Biotherapies, personal communication).

\section{Statistical analysis}

For each quarter the proportion of new clients with a genital wart diagnosis was calculated by dividing the number of genital wart diagnoses by the number of new clients seen. These proportions were modelled using logistic regression with separate linear trends (on the logit scale) fitted for two time periods, Q1 2004 to Q4 2007, and Q1 2008 to Q4 2008. The choice of change-point to define the two periods was based on the assumption that before 2008 there were very few people who had been fully immunised with three doses of the vaccine.
For each time period the estimated increase in log odds of the proportion of new clients with genital warts was converted to a percentage change, which is presented with 95\% CI. The estimated pre and post-Q4 2007 trends were compared using a Wald test. We examined the robustness of the assumption of linear trend by fitting models with an additional quadratic term in each time period and comparing this with the corresponding model with only a linear trend, also using a Wald test. Analyses were stratified by gender and age (for women), and for men they were further stratified by the sex of their partners in the past 12 months.

We calculated percentage change in the odds of genital warts from the fitted logistic regression models by exponentiating (anti-logging) the relevant estimated regression coefficient (giving an odds ratio for a unit change in exposure), submitting the null value 1.00 (corresponding to no change) and multiplying by 100. As the prevalence of genitals warts is low (less than $10 \%$ in all subgroups) the percentage change in odds will be similar to the percentage change in proportion; we report the latter throughout the study.

All calculations were performed using Stata version 10.1. The study was approved by the Alfred Hospital Ethics Committee as involving "negligible risk" and did not require a formal ethics submission.

\section{RESULTS}

Between 1 January 2004 and 31 December 2008, 36055 clients attended MSHC for the first time and genital warts were diagnosed in 3826 (10.6\%; 95\% CI 10.3 to 10.9). The number of clients diagnosed with warts by year and risk group is shown in table1 and the estimated trends for the two time periods are shown in table 2 . Only women under 28 years of age showed strong evidence of a significant difference in the average quarterly change between the two time periods $(1.8 \%$ increase before end-2007 vs $25.1 \%$ decrease after end-2007, p<0.001). For

Table 2 Average percentage change per quarter in the proportion of patients presenting with genital warts (with $95 \% \mathrm{CI}$ ) for two time periods, 01 2004 to 042007 and 012008 to 042008

\begin{tabular}{|c|c|c|c|c|c|}
\hline & $\begin{array}{l}\text { Change per quarter before } \\
\text { end } 2007(95 \% \mathrm{CI})\end{array}$ & p Value & $\begin{array}{l}\text { Change per quarter after } \\
\text { end } 2007(95 \% \mathrm{CI})\end{array}$ & p Value & $\begin{array}{l}\text { p Value comparing change } \\
\text { per quarter before and } \\
\text { after end } 2007\end{array}$ \\
\hline Women $<28$ years & $+1.8 \%(+0.2 \%$ to $+3.4 \%)$ & 0.03 & $-25.1 \%(-30.5 \%$ to $-19.3 \%)$ & $<0.001$ & $<0.001$ \\
\hline Women $\geqslant 28$ years & $+1.0 \%(-1.5 \%$ to $+3.7 \%)$ & 0.43 & $-4.7 \%(-13.9 \%$ to $+5.4 \%)$ & 0.34 & 0.32 \\
\hline Men sex with men only* & $+0.5 \%(-2.1 \%$ to $+3.1 \%)$ & 0.71 & $-5.9 \%(-14.6 \%$ to $+3.5 \%)$ & 0.21 & 0.25 \\
\hline Men sex with women only* & $-0.6 \%(-1.8 \%$ to $+0.6 \%)$ & 0.31 & $-5.0 \%(-9.4 \%$ to $-0.5 \%)$ & 0.03 & 0.10 \\
\hline Primary genital herpes & $+1.8 \%(-0.1 \%$ to $+3.8 \%)$ & 0.06 & $-3.4 \%(-9.9 \%$ to $+3.4 \%)$ & 0.32 & 0.20 \\
\hline
\end{tabular}

A positive percentage indicates a quarter-to-quarter increase, a negative percentage indicates a quarter-to-quarter decrease. $p$ Values are presented for the null hypotheses of no quarterly change within each period and for the null hypothesis that the quarterly change before and after end-2007 is the same. ${ }^{*}$ Refers to the gender of the sexual partners in the past 12 months. 
Presentations with Warts

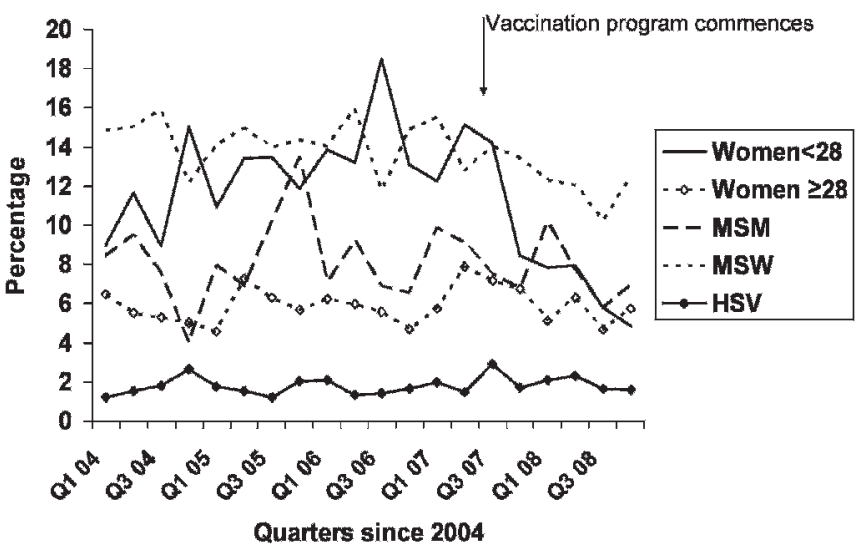

Figure 1 Presentations with warts. HSV, herpes simplex virus (primary genital herpes); MSM, men who have had sex with men but not women in the past year; MSW, men who have had sex with women but not men in the past year. Arrow refers to the commencement of the free vaccination programme against human papillomavirus.

this group (women $<28$ years of age) there was some curvature in the relationship between the log odds of diagnosis with genital warts (in the logistic regression model, the linear coefficient for time period 1 was $-0.0612,95 \%$ CI -0.1203 to -0.0022 and the quadratic coefficient was $-0.0057,95 \%$ CI -0.0098 to -0.0016 , with the time variable taking value 1 in quarter 1, 2004). The degree of curvature after accounting for the linear trend was, however, small and therefore the resulting trend was captured adequately using a linear term in the model.

There was no evidence of a difference in trend for the quarterly proportions before and after the end of 2007 for any other subgroup, and for only one subgroup was there strong evidence of a trend different to zero, for heterosexual men in 2008 when the average quarterly change was a decrease of $5 \%$ (95\% CI $0.5 \%$ to $9.4 \%$; $\mathrm{p}=0.031$ ).

Of note, the number of male sexual partners among women less than 28 years of age in 2004-7 and 2008 did not differ (mean of 2.5 and 2.4, respectively; $p=0.19$ ), and the proportion of clients attending MSHC diagnosed with herpes for the first time was stable during the study period (table 1, fig 1).

\section{DISCUSSION}

We saw a marked reduction in clinical presentations for genital warts among women in the target age group for HPV vaccination in the year following the implementation of a national HPV vaccination programme; a fall that clearly diverged from previous trends in clinical diagnoses. The only other subgroup to have a modest fall in presentations for genital warts was heterosexual men in 2008. Notably, there was no difference in genital wart diagnoses between the two time periods (2004-7 and 2008) or any reduction observed in 2008 among homosexual men or women outside the age group eligible for free vaccination. The magnitude of the reduction in women less than 28 years indicates a potential for substantial reductions in wart-associated morbidity and costs and has important implications for countries deciding between the bivalent and quadrivalent vaccine..$^{13}$ Our findings are consistent with, and provide strong, plausible evidence for, the effectiveness of the vaccine at the population level. Clinical trials have

\section{Key messages}

- Australia began a free quadrivalent HPV vaccination programme in mid-2007 for schoolgirls and women under 28 years of age, with coverage of approximately $70 \%$.

- Since 2008 there has been a substantial and significant decline in genital warts in women 28 years of age and younger, but not in women over 28 years of age at the sexual health clinic.

- Since 2008 there has been a significant decline in genital warts in heterosexual but not homosexual men.

clearly demonstrated the efficacy in vaccinated individuals but not the effectiveness at a population level.

Our study has a number of limitations. First, the data came from a single clinic, albeit, Australia's largest sexual health clinic and one that diagnoses up to $25 \%$ of the state's notifiable sexually transmitted infections. It would be difficult to obtain representative data from entire communities because genital warts are not generally notified by national surveillance systems in Australia or in most other countries. Second, it is possible that unmeasured confounding may have explained the observed changes. Several pieces of evidence, however, point to our observations not being confounded by sexual risk. These include the absence of any change in initial diagnoses of genital herpes and consistency in the number of sexual partners reported for women less than 28 years attending the centre over the study period, and finally the magnitude of the change seen in the group eligible for free vaccination. Further evidence of a real effect of vaccination is the fall in the absolute number of warts in women less than 28 years rather than just the proportion. Importantly, there were no changes in clinic policy either relating to the selection of clients attending the centre or clinical management and diagnosis, which would account for the observed reductions in genital warts. Comprehensive data on the prevalence of cervical dysplasia in women attending this clinical service over the study period was not available for analysis.

We did not have data on whether individuals attending the centre had or had not received the HPV vaccine. However, the aims of our study were to assess the population-level effects of vaccination rather than the efficacy of the vaccine, which has been well established in randomised clinical trials.

Our data suggest that a relatively rapid and marked reduction in the population prevalence of genital warts among vaccinated women may be achievable through an HPV vaccination programme targeting women. Our data also supports some potential benefit being conferred to men. The reduction in genital wart diagnoses in 2008 among heterosexual but not among homosexual men may be consistent with reduced heterosexual transmission of HPV as a result of female vaccination. These data should be of value to governments making decisions about whether to implement HPV vaccination in women and may assist in informing the choice of vaccine. Surveillance of genital warts should be compared in settings and countries where HPV vaccination has been implemented using bivalent and quadrivalent vaccines to determine the degree to which the quadrivalent vaccine reduces the prevalence of genital warts at a community level.

Acknowledgements: The authors would like to thank Afrizal Afrizal and Jun Kit Sze for the extraction of the data from MSHC. The authors are also grateful to $\mathrm{Dr}$ Rosemary Lester from the Department of Human Services and Mr Greg Whiteside from CSL Biotherapies for the vaccination coverage data. 
Funding: CSB holds a National Medical and Research Council Research Fellowship, grant ID 465164. JSH and LCG hold National Health and Medical Research Council Career Development awards ID 566576 and 454779. No funding was obtained to undertake this study.

Competing interests: Declared. CKF owns shares in CSL Biotherapies, the manufacturer of Gardasil. CKF, JSH and BD have received honoraria from CSL Biotherapies. BD has received honoraria from Glaxo SmithKline and BD and CKF have received research funding from CSL Biotherapies. MYC, LCG and CSB have no conflicts of interest.

Ethics approval: The study was approved by the Alfred Hospital Ethics Committee as involving "negligible risk" and did not require a formal ethics submission.

Contributors: CKF, JSH, MYC, BD and CSB conceived and designed the study. CKF, JSH and LCG undertook the analysis. All authors critically revised the article for intellectual content and approved the final version. CKF has full access to all the data in the study and takes responsibility for the integrity of the data and the accuracy of the data analysis.

Provenance and peer review: Not commissioned; externally peer reviewed.

\section{REFERENCES}

1. Villa L, Costa RL, Petta CA, et al. Prophylactic quadrivalent human papillomavirus (types 6, 11, 16 and 18) L1 virus like particle vaccine in young women: a randomised double blind placebo controlled multi centre phase 11 efficacy trial. Lancet Oncol 2005:6:271-8.

2. Koutsky L, Ault KA, Wheeler CM, et al. A controlled trial of human papillomavirs type 16 vaccine. N Engl J Med 2002;347:1645-51.
3. Mao C, Koutsky LA, Ault KA, et al. Efficacy of human papillomavirus-16 vaccine to prevent cervical intraepithelial neoplasia - a randomised controlled trial. Obstet Gynecol 2006;107:18-27.

4. Harper D, Franco EL, Wheeler CM, et al. Sustained efficacy up to 4-5 years of a bivalent $\mathrm{L} 1$ virus-like particle vaccine against human papillomavirus types 16 and 18 : a follow -up from a randomised controlled trial. Lancet 2006;367:1247-55.

5. Villa L, Ault KA, Giuliano AR, et al. Immunologic responses following administration of a vaccine targeting human papillomavirus types $6,11,16$ and 18. Vaccine 2006:24:5571-83.

6. Villa L, Costa RL, Petta CA, et al. high sustained efficacy of a prophylactic quadrivalent human papillomavirus types 6/11/16/18 L1 virus-like particle vaccine through 5 years of follow up. Br J Cancer 2006;95:1459-66.

7. The Future II Study Group. Quadrivalent vaccine against human papillomavirus to prevent high grade cervical lesions. N Engl J Med 2007;356:1915-27.

8. Barr E, Tamms G. Quadrivalent human papilloma vaccine. Clin Infect Dis 2007:45:609-17.

9. Giuliano A, Palefsky J. The efficacy of quadrivalent HPV (types 6/11/16/18) vaccine in reducing the incidence of HPV infection and HPV-related genital disease in young men [abstract (SS19-197a)]. EUROGIN 2008 Congress: Joining Forces for Cervical Cancer Prevention; Nice, 2008. http://www.eurogin.com/2008/ EUROGIN2008 LastMinuteAbstracts.pdf (accessed: 15 Sept 2009).

10. Kulasingam S, Connelly L, Conway E, et al. A cost-effectiveness analysis of adding a human papillomavirus vaccine to the Australian National Cervical Cancer Screening Program. Sexual Health 2007:4:165-75.

11. Jit M, Choi YH, Edmunds WJ. Economic evaluation of human papillomvirus vaccination in the United Kingdom. BMJ 2008:337:a769.

12. Tideman RL, Pitts MK, Fairley CK. Effects of a change from an appointment service to a walk-in triage service at a sexual health centre. Int J STD AIDS 2003;14:793-5

13. Hu D, Goldie S. The economic burden of noncervical human papillomavirus disease in the United States. Am J Obstet Gynecol 2008;198:e1-7. 